\title{
Persistent Bioperl
}

BOSC 2003

Hilmar Lapp

Genomics Institute of

The Novartis Research Foundation (GNF) 


\section{Acknowledgements}

Bio* contributors and core developers

Aaron, Ewan, ThomasD, Matthew, Mark, Elia, ChrisM, BradC, Jeff Chang, Toshiaki Katayama

And many others

Sponsors of Biohackathons

Apple (Singapore 2003)

O' Reilly (Tucson 2002)

Electric Genetics (Cape Town 2002)

GNF for its generous support of OSS development 


\section{Overview}

\section{Use cases \\ BioSQL Schema \\ Bioperl-DB \\ Key features and design goals \\ Examples}

Status \& Plans

Summary 


\section{Use cases (I)}

' Local GenBank with random access'

Local cache or replication of public databanks

Indexed random access, easy retrieval

Preserves annotation (features, dbxrefs,...), possibly even format

' GenBank in relational format'

Normalized schema, predictably populated

Allows arbitrary queries

Allows tables to be added to support my data/question/... 


\section{Use Cases (II)}

' Integrate GenBank, Swiss-Prot, LocusLink, ...'

Unifying relational schema

Provide common (abstracted) view on different sources of annotated genes

- Database for my lab sequences and my annotation' Store FASTA-formatted sequences Add, update, modify, remove various types of annotation 


\section{Use Cases (III)}

Persistent storage for my favorite Bio* toolkit Relational model accommodates object model Persistence API with transparent insert, update, delete 


\section{Persistent Bio*}

Normalized relational schema designed for Bio* interoperability

BioSQL

Toolkit-specific persistence API

Bioperl-DB 


\section{BioSQL}

Interoperable relational data store for Bio*

Language bindings presently for Bioperl, Biojava, Biopython, Bioruby

Very flexible, normalized, ontology-driven schema

Focal entities are Bioentry, Seqfeature, Term (and Dbxref)

Schema instantiation scripts for different RDBMSs

MySQL, PostgreSQL, Oracle

Release of v1.0 imminent

Schema has been stable for the last 3 months

Relatively well documented (installation, how-to, ERD)

Mailing list (biosql-I@open-bio.org), CVS (biosql-schema), links at http://obda.open-bio.org 


\section{BioSQL: Some History}

Ewan Birney started BioSQL and Bioperl-db in Nov 2001 Initial use-case was to serialize/de-serialize Bio::Seq objects to/from a local sequence store (as a replacement for SRS) Schema redesigned at the 2002 Biohackathons in Tucson and Cape Town

Series of incremental changes later in 2002

Full review at the 2003 Biohackathon in Singapore

Changed Taxon model to follow NCBl' $s$

Full ontology model, resembles GO' s model

Features can have dbxrefs

Consistent naming 


\section{BioSQL ERD}

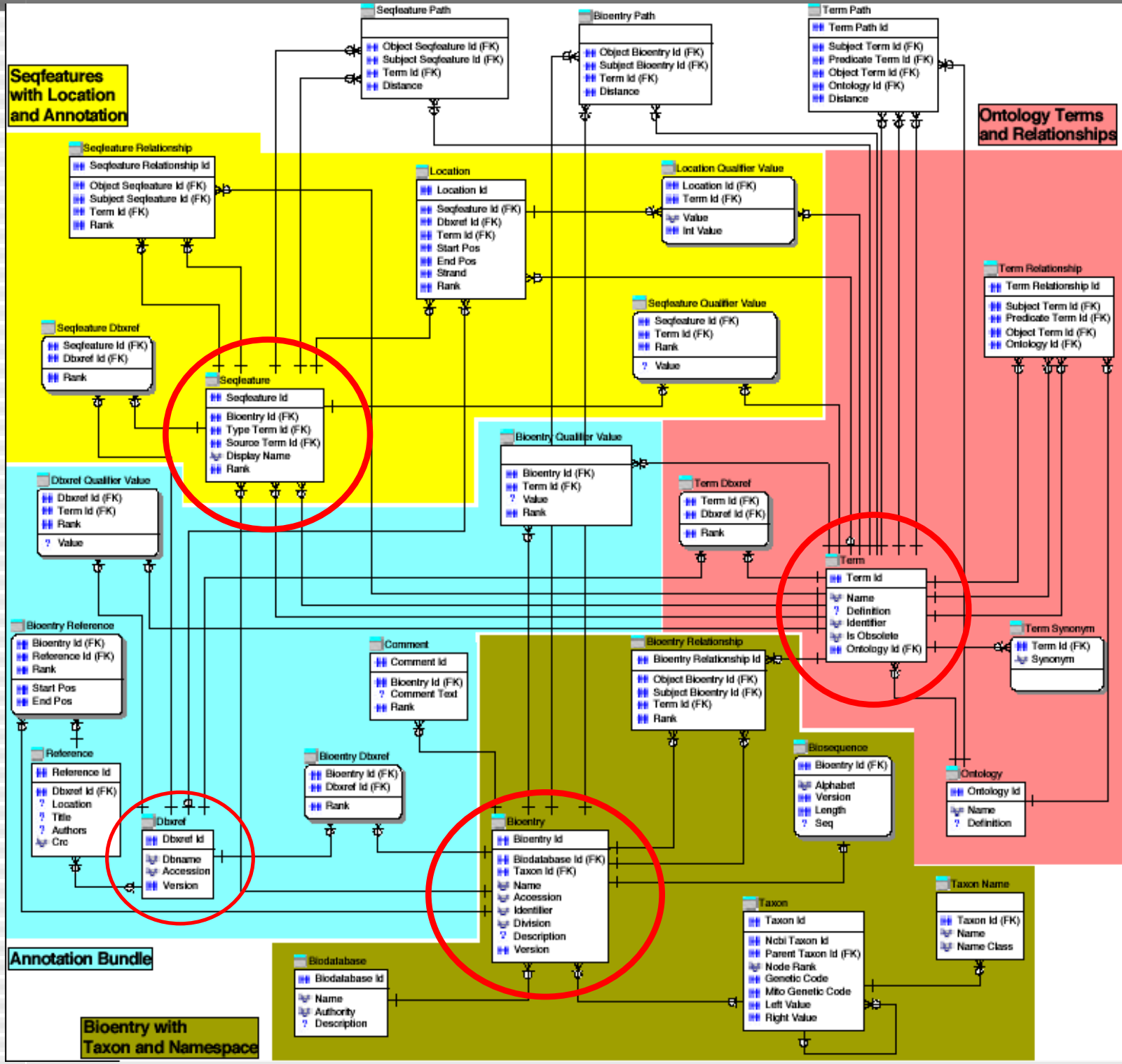




\section{BioSQL ERD}

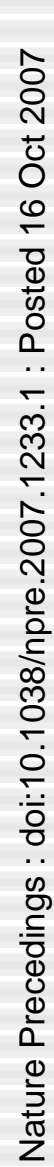

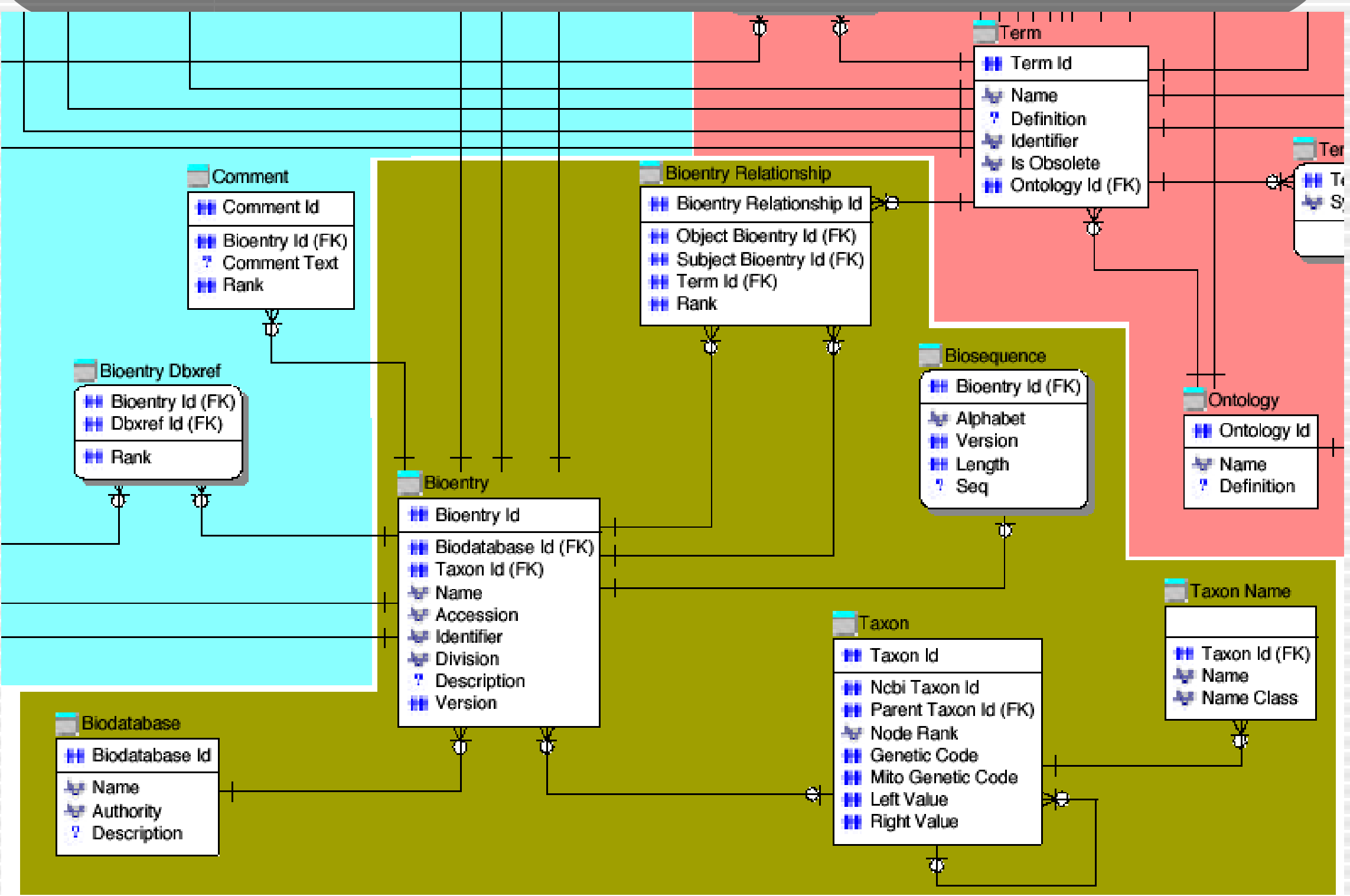




\section{BioSQL ERD}

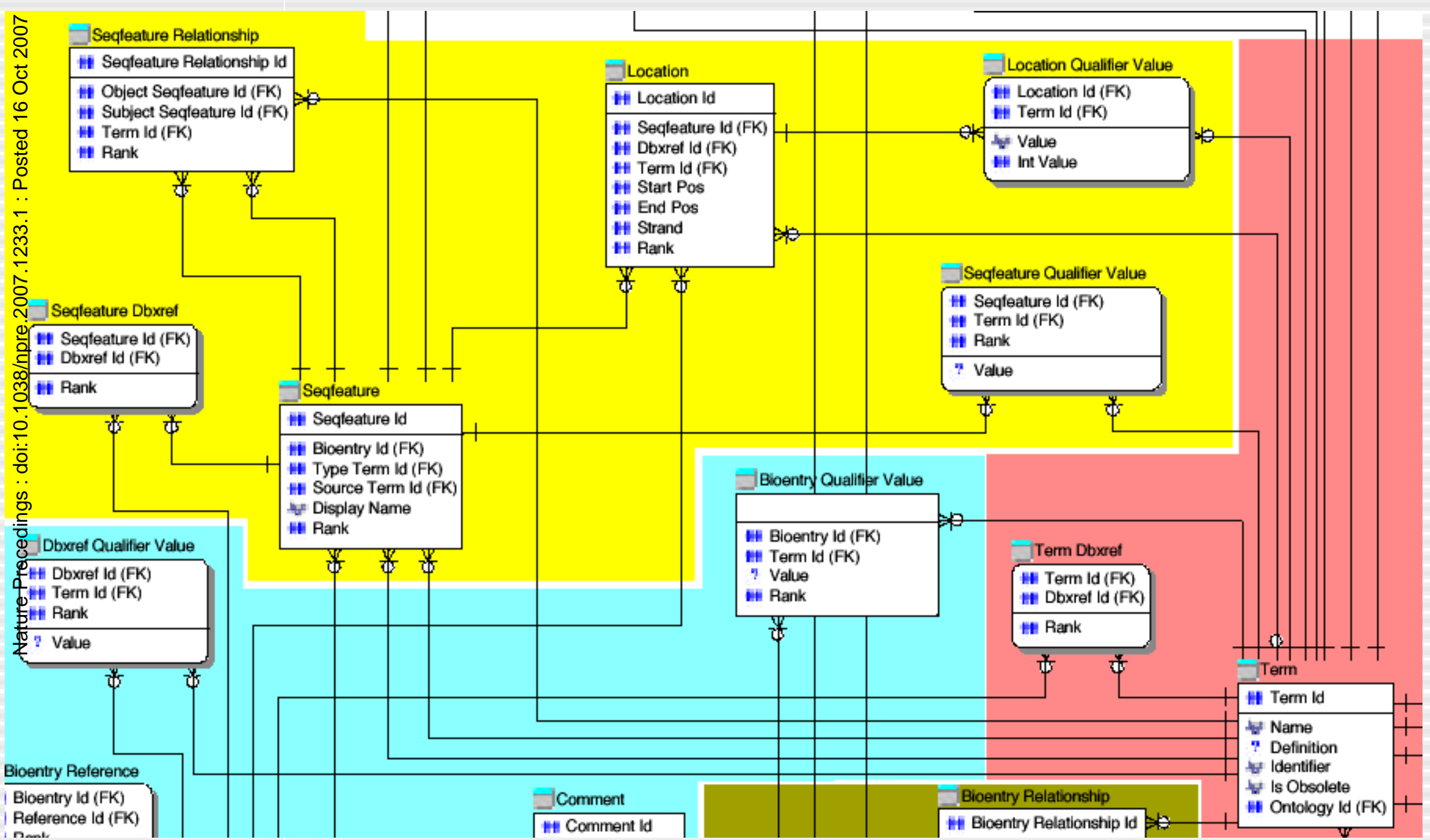




\section{BioSQL ERD}

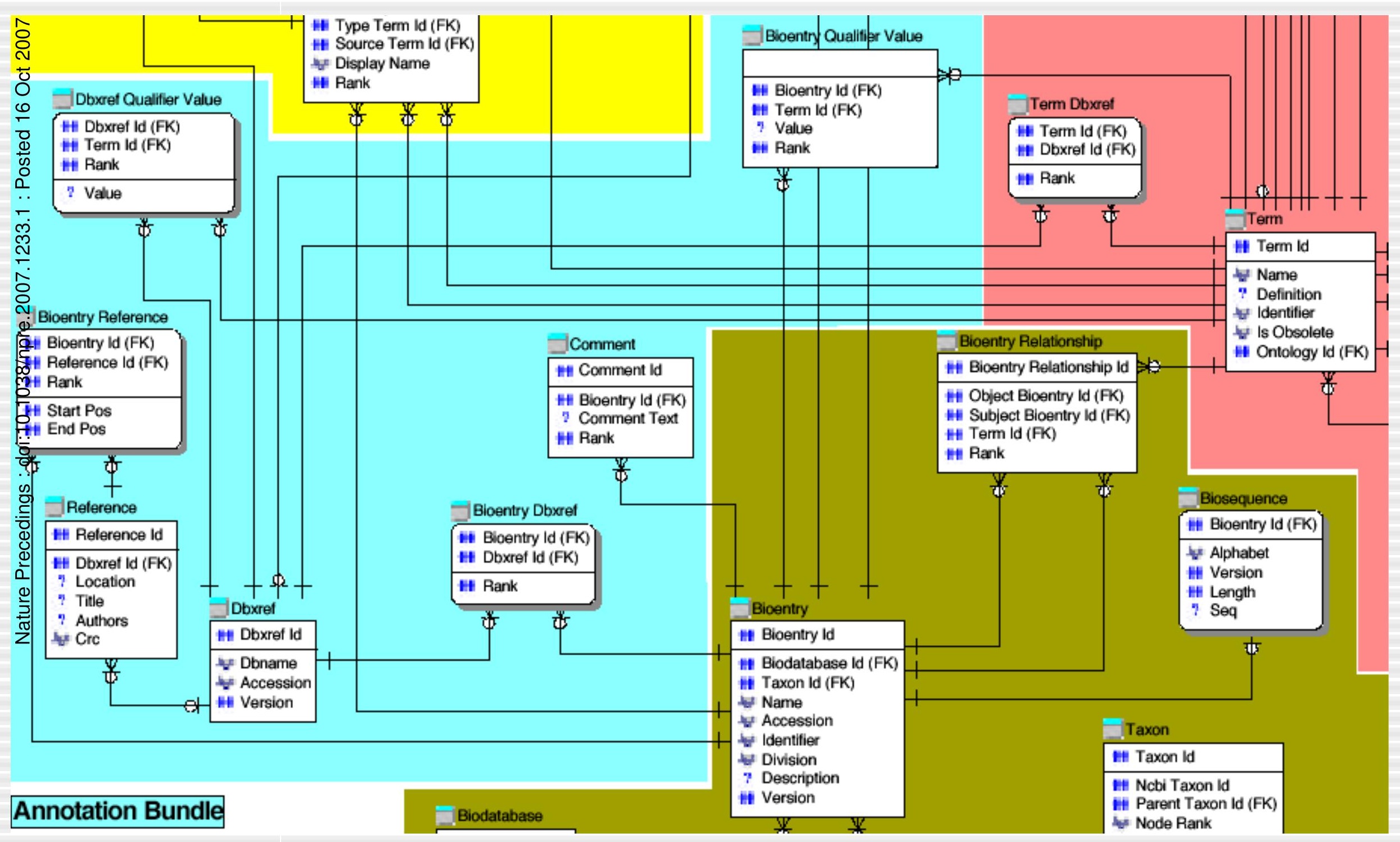




\section{BioSQL ERD}

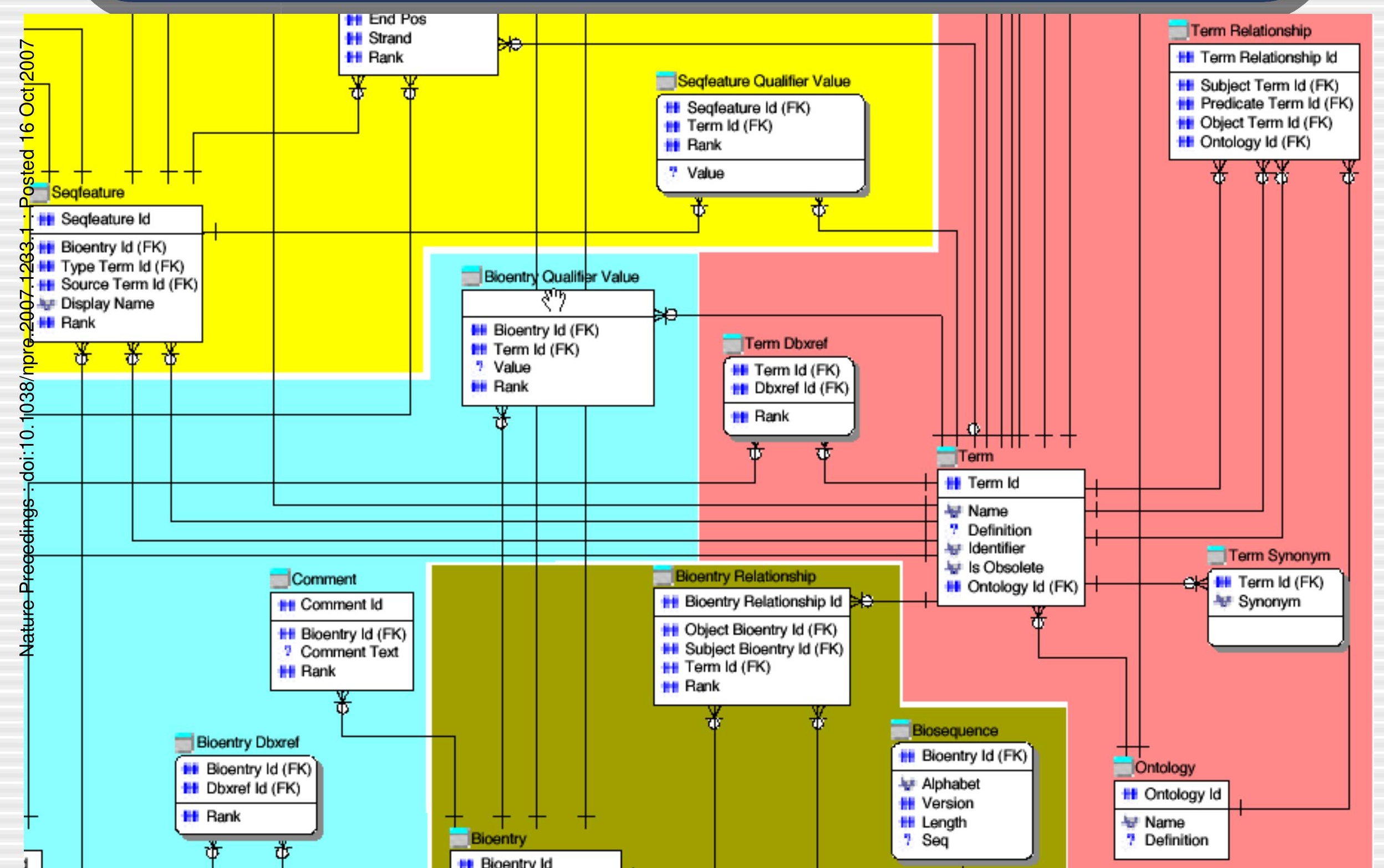




\section{Language Binding: OR Mapping}

Object-Relational Mapping connects two worlds

Object model (Bioperl) $\leftrightarrow$ Relational model (Biosql)

Object and relational models are orthogonal (though

' correlated' )

- E.g., inheritance, n:n associations, navigability of associations, joins

General goals of the OR mapping are

Bi-directional map between objects and entities

Transparent persistence interface reflecting all of INSERT, UPDATE, DELETE, SELECT

Generic approaches exist, most of which are commercial

TopLink, CMP (e.g., Jboss), JDO, Tangram 


\section{Bioperl-db Is An OR-Mapper}

\# get persistence adaptor factory for database my $\$ d b=$ Bio::DB::BioDB->new (-database $\Rightarrow$ 'biosql', -dbcontext $\Rightarrow$ \$dbc);

\# open stream of objects parsed from flatfile my stream = Bio: :SeqIO->new (-fh $\quad \Rightarrow \mid * S T D I N$, -format $\Rightarrow$ ' genbank');

while(my \$seq = \$stream->next_seq())

\# convert to persistent object \$pobj = \$db->create_persistent (\$seq);

\# insert into datastore \$pobj->create () ; 


\section{Where can I get Bioperl-db?}

Bioperl-db is a sub-project of Bioperl

Links and news at http://www.bioperl.org/

Email to bioperl-|@bioperl.org

- but biosql-|@open-bio.org will often work, too

CVS repository is bioperl-db under bioperl

(/home/repository/bioperl/bioperl-db)

No release of the current codebase yet

But v0.2 is imminent 


\section{Bioperl-db: Key Features (I)}

Transparent persistence API on top of object API

Persistent objects know their primary keys, can update, insert, and delete themselves

- Full API in Bio::DB::PersistentObjectl

Peristent objects speak both the persistence API and their native tongue

Several retrieval methods on the persistence adaptor API: find_by_primary_key(), find_by_unique_key(), find_by_query(), find_by_association()

Full API in Bio::DB::PersistenceAdaptorl 


\section{Bioperl-olb: Key Features (II)}

Extensible framework separating object adaptor logic from schema logic

Central factory loads and instantiates a datastore-specific adaptor factory at runtime.

Adaptor factory loads and instantiates persistence adaptor at runtime - no hard-coded adaptor names

Queries are constructed in object space and translated to SQL at run-time by schema driver

Designed with adding bindings to other schemas than BioSQL in mind (e.g., Chado, Ensembl, MyBioSQL, ...) 


\section{Bioperl-db: Examples (I)}

\section{Step 1: connect and obtain adaptor factory}

use Bio::DB: :BioDB;

\# create the database-specific adaptor factory

\# (implements Bio::DB::DBAdaptorI)

$\$ d b=$ Bio: :DB: :BioDB->new (-database =>"biosql",

\# user, pwd, driver, host ...

- dbcontext $=>$ \$dbc); 


\section{Bioperl-db: Examples (II)}

- Step 2: depends on use case Load sequences:

use Bio: : SeqIO;

\# open stream of objects parsed from flatfile my $\$$ stream = Bio: : SeqIO->new $(-\mathrm{fh}=>\backslash * \operatorname{STDIN}$, -format $=>$ ' genbank') ;

while(my \$seq = \$stream->next_seq())

\# convert to persistent object \$pseq $=$ \$db->create_persistent (\$seq);

\# \$pseq now implements Bio::DB::Persistentobject I

\# in addition to what \$seq implemented before

\# insert into datastore \$pseq->create () ; 


\section{Bioperl-db: Examples (III)}

- Step 2: depends on use case Retrieve sequences by alternative key:

use Bio::Seq; use Bio:: Seq: :SeqFactory; \# set up seq object as query template \$seq = Bio: :Seq->new (-accession_number => "NM_000149",

-namespace => "RefSeq");

\# pass a factory to leave the template object untouched \$seqfact = Bio: : Seq: :SeqFactory->new (-type=> "Bio:: Seq"); \# obtain object adaptor to query (class name works too) \# adaptors implement Bio::DB::PersistenceAdaptorI \$adp = \$db->get_object_adaptor (\$seq);

\# execute query

\$dbseq $=$ \$adp->find_by_unique_key ( \$seq, -obj_factory $=>$ \$seqfact);

warn \$seq->accession_number(),

" not found in namespace RefSeq $\backslash n$ " unless \$dbseq; 


\section{Bioperl-db: Examples (IV)}

- Step 2: depends on use case Retrieve sequences by query:

use Bio::DB: : Query: :BioQuery;

\# set up query object as query template \$query = Bio: :DB: : Query: :BioQuery->new (

-datacollections $=>$ ["Bio:: Seq $s "$ ",

"Bio: : Species=>Bio: : Seq sp"],

-where $\quad=>$ ["S.description like 'okinase\%" ",

"sp.binomial = ?"]);

\# obtain object adaptor to query

\$adp $=$ \$db->get_object_adaptor("Bio: :SeqI") ;

\# execute query

\$qres = \$adp->find_by_query (\$query, -name => "bosc03", -values $=>$ ["Homo sapiens"]);

\# loop over result set

while(my \$pseq = \$qres->next_object ()) \{ print \$pseq->accession_number, "\n"; 


\section{Bioperl-db: Examples (V)}

- Step 2: depends on use case

Retrieve sequence, add annotation, update in the $\mathrm{db}$

use Bio:: Seq; use Bio: : SeqFeature: Generic;

\# retrieve the sequence object somehow ...

\$adp $=$ \$db->get_object_adaptor ("Bio: :SeqI") ;

\$dbseq = \$adp->find_by_unique_key (

Bio: :Seq->new (-accession_number => "NM_000149",

-namespace $\quad=>$ "RefSeq"));

\# create a feature as new annotation

\$feat = Bio: : SeqFeature: :Generic->new (

-primary_tag $=>$ "TFBS",

-source_tag $=>$ "My Lab",

- start $=>23$, - end $=>27$, - strand $=>-1$ ) ;

\# add new annotation to the sequence

\$dbseq->add_SeqFeature (\$feat);

\# update in the database

\$dbseq->store () ; 


\section{Bioperl-db: Examples (Vla)}

- Extensibility: handle my own object by adding my own adaptor.

\section{A) Custom sequence class}

package MyLab: :Y2HSeq;

QISA = qw (Bio: :Seq);

sub get_interactors \{

my self = shift;

return @ $\{\$$ self $->\{$ '_interactors' $\}\}$;

\}

sub add_interactor \{

my $\$$ self $=$ shift;

push (@ $\{\$ \operatorname{sel} f->\{$ '_interactors' $\}\}$, Q_) ;

\}

sub remove_interactors \{

my $\$$ self $=$ shift;

my darr = \$self->get_interactors ();

\$self->\{'_interactors' $\}=$ [ ];

return earr; 


\section{Bioperl-db: Examples (Vlb)}

- Extensibility: handle my own object by adding my own adaptor. B) Custom adaptor class

package Bio::DB::BioSQL: :Y2HSeqAdaptor; QISA = qW(Bio::DB::BioSQL: : SeqAdaptor); sub store_children \{

my $(\$ s e l f, \$ o b j)=d_{-}$;

\# call inherited method

\$self->SUPER: : store_children (d_);

\# obtain persistent term object for the rel.ship type my \$term = Bio:: Ontology:: Term->new (

-name $=>$ "interacts-with",

-ontology $=>$ "Relationship Types");

my $\$$ termadp $=\$$ self->db->get_object_adaptor (\$term);

my \$reltype = \$termadp->find_by_unique_key (\$term) or

\$self->db->create_persistent (\$term)->create ();

\# continued on the next page ... 


\section{Bioperl-db: Examples (Vlb)}

- Extensibility: handle my own object by adding my own adaptor. B) Custom adaptor class (cont' d)

\# store the interacting sequences

foreach my \$seq (\$obj->get_interactors()) \{

\# each interactor needs to be persistent object \$seq $=$ \$self->db->create_persistent (\$seq)

unless \$seq->isa ("Bio::DB::PersistentobjectI");

\# each interactor also needs to have a primary key \$seq $=$ \$seq->adaptor->find_by_unique_key() or

\$seq->create ();

\# associate the interactor with this object \$seq->adaptor->add_association (

-objs $=>$ [\$obj, \$seq, \$reltype],

-contexts $=>$ ["object","subject", undef]);

\}

return 1; \# done 


\section{Ready-To-Use Scripts (I)}

load_seqdatabase.pl (bioperl-db/scripts/biosql)

Use for loading and updating bioentries and their annotation

Supports all Bio::SeqlO supported formats

- genbank, embl, swiss, locuslink, fasta, gcg, ace, ...

Supports all Bio::ClusterlO supported formats

- Unigene

Many command line options

For flexible handling of updates

- --lookup, --noupdate, --remove, --mergeobjs

For filtering and post-processing sequences

- --seqfilter, --pipeline 


\section{Ready-To-Use Scripts (II)}

- load_ontology.pl (bioperl-db/scripts/biosql)

Use for loading and updating ontologies and terms

Supports all Bio::OntologylO supported formats

- dagflat (incl. soflat, goflat), InterPro, simplehierarchy

Tested for GO and SOFA

Many command-line options

For handling updates and obsoleted terms

- -lookup, --noupdate, --remove

- --noobsolete, --updobsolete, --delobsolete, --mergeobjs

For (re-)computing the transitive closure

- --computetc 


\section{Ready-To-Use Scripts (III)}

- load_ncbi_taxonomy.pl (biosql-schema/scripts)

Use for loading and updating the taxon tables with the NCBI Taxonomy database

Downloads the database from NCBI automatically if desired Some options to configure and tune load and update Automatically updates the Nested Set values in the taxon table 


\section{Current Status}

BioSQL is stable and release-ready

Imminent release of $\mathrm{v} 1.0$

Well-documented ;-) , ER-diagram

Supports MySQL, PostgreSQL, and Oracle

Toolkit-independent script for populating taxa

Bioperl-db is stable but documentation is patchy

Core APIs stable and documented, but no How-To' s

All tests pass on all 3 RDBMS platforms

Head revision wants Bioperl >=1.2.2 (but for RichSeql

attributes you need Bioperl main trunk)

Fuzzy locations get transformed to simple locations

BioSQL \& Bioperl-db are used in production and at multiple places 


\section{Plans For The Future (I)}

Persistence Adaptors for more object types

Phenotypes (OMIM)

Markers (SNPs, STSs, ...)

Increased support for lazy loading

Features and annotations for a sequence (sequence itself is already lazy-loaded)

Write adaptors for other applications to run off of BioSQL

Genome browsers: GBrowse, Apollo

Ontology editors: DAG-edit 


\section{Plans For The Future (II)}

Proof-of-Concept for interoperability

Load through Bioper//Bioperl-db, retrieve through Biojava

Proof-of-Concept of the architecture' s flexibility

Map to schemas different from BioSQL: Chado, Ensembl 


\section{Summary}

- BioSQL is a very flexible, ontology-driven, stable relational schema to capture richly annotated databank entries

BioSQL is supported as the persistent storage across the Bio* projects

Bioperl-db is the object-relational mapping for Bioperl objects to BioSQL

Bioperl-db adds a transparent persistence API on top of all supported Bioperl objects

- Presently supported areas of the object model are sequences, features, annotations, clusters, ontologies 